\title{
A SIMULATOR-BASED STREET-CROSSING TRAINING FOR OLDER PEDESTRIANS: SHORT AND LONG TERM EFFECTS
}

\author{
Aurélie Dommes \& Viola Cavallo \\ French National Institute for Transport and Safety Research \\ Laboratory of Driver Psychology \\ Arcueil, France \\ Email: dommes@inrets.fr; cavallo@inrets.fr
}

\begin{abstract}
Summary: The study aimed at developing and assessing a training method to improve the safety of elderly pedestrians with a simulator-based street-crossing technique specially designed to their needs and difficulties. Twenty seniors were enrolled in a street-crossing training program, and twenty other seniors were assigned to the control group (internet-use training). Before the training, immediately after it, and 6 months later, street-crossing decisions and behaviors were assessed using a simulated street-crossing task. The results showed that the simulator-based training enhanced the safety of the elderly pedestrians. However, the way in which they took into account the speed of the approaching car in their decisions and behaviors had not improved. The lack of effectiveness of training in the use of speed may reveal age-related sensory and cognitive impairments that our simulator-based method could not alleviate. The results of this study stressed the importance of greater attention to senior street-crossing retraining.
\end{abstract}

\section{INTRODUCTION}

International accident statistics indicate that elderly pedestrians are an extremely vulnerable road-user group (NHTSA, 2001). In France, more than half of all pedestrians killed on the road $(51 \%)$ are over 65 years old, whereas this age group represents less than $15 \%$ of the population (ONISIR, 2006). Psychology research (e.g. Lobjois \& Cavallo, 2007; Oxley, Fildes, Ihsen, Charlton, \& Day, 2005) has highlighted some of the age-specific characteristics of road-crossing behavior: slowing of decision-making, decreased walking speed, and difficulty in selecting safe gaps and adopting sufficient safety margins. More specifically, these studies demonstrated an effect of the approaching car's speed on elderly adults' decisions: contrary to younger adults, who accepted constant time gaps, older pedestrians were found to accept shorter and shorter time gaps as the car's speed increased, putting them at a higher risk at high speeds. They also tended to miss many safe opportunities to cross in front of cars approaching at low speeds.

Although for many seniors, pedestrian travel is the major way of getting around, surprisingly, there doesn't seem to be any research that focused on designing a training program to improve the safety of older pedestrians. The present study was aimed at contributing to this issue. The objective was to develop and assess a training method to enhance the safety of elderly pedestrians, a method specially designed with their needs and difficulties.

A new research trend on retraining older adults in the perceptual, motor, and/or cognitive skills of daily activities in view of maintaining their autonomy and improving their quality of life emerged about twenty years ago and has been growing ever since. Data from laboratory studies 
clearly indicate that elderly adults can improve their performance with practice and training. Some studies have focused on older drivers and how they handle their difficulties. In a systematic review of the literature, Kua, Korner-Bitensky, Desrosiers, Man-Son-Hing, and Marshall (2007) assessed the effectiveness of retraining programs for older drivers, while making the distinction between physical retraining (e.g. at-home stretching exercises of the upper body: Ostrow, Shaffron, \& McPherson, 1992), visual perception programs (e.g., speed-ofprocessing retraining: Roenker, Cissel, Ball, Wadley, \& Edwards, 2003), educational programs (educational curriculum: Owsley, Stalvey, \& Phillips, 2003), and mixed interventions (e.g., physical, perceptual, and educational programs: McCoy, Tarawneh, Bishu, Ashman, \& Foster, 1993). The available findings are scarce, but encouraging enough to promote further investigation in elderly-driver retraining.

Whereas street-crossing trainings have been developed for children (i.e. skill learning), interestingly, no study has explored the benefits of a physical, perceptive, or educational retraining intervention to enhance the safety of older pedestrians. The present study was intended to contribute to this issue. The method specifically addresses rehabilitation of the behavioral component of street crossing by providing simulator-based street-crossing training. Older pedestrians are trained on an interactive street-crossing simulator which offers not only a good level of validity (Cavallo, Lobjois, \& Vienne, 2006), but allows a safe street-crossing as well as a perfect control of the characteristics of the traffic. The training method promotes individual sensory-motor practice and addresses elderly adults' ways of thinking about the task and the strategies they bring into play. Through feedback, the training is aimed at modifying older adults' street-crossing strategies. More specifically, one of our objectives is to improve the way seniors process the speed of an approaching car and use it in their decisions and behaviors. In sum, by means of repeated practice and a better understanding of the task constraints, the training program is aimed at improving the overall safety of elderly adults when crossing the street, and also at helping them take the approaching car's speed into account in a safer way.

\section{METHOD}

\section{Participants}

Twenty elderly participants (11 women and 9 men, mean 73.05, SD 4.4) were enrolled in the street-crossing training program. A control group of 20 elderly participants (12 women and 8 men, mean 71.4, SD 5.8) was pre- and post-tested at the same time as were participants in the experimental group. Instead of the street-crossing training, the control group participants were given an internet-use training course. All participants obtained scores on the Mini Mental State Examination over 27 (mean 28.9, SD 0.9), indicating they were not affected by pathologic aging.

\section{Experimental Setup}

The street-crossing simulation device was based on the INRETS Sim2 driving simulator (Espié, 1999) adapted to the street-crossing situation (Cavallo et al., 2006). The device included a portion of experimental road (4.2-m wide, materialized on the ground), an image-generation system, three-screen projection $(2.70 \times 1.90 \mathrm{~m})$, a 3D sound-rendition system, and a recording system. The setup provided the participant with a horizontal visual field between $90^{\circ}$ (at the 
starting point) and $140^{\circ}$ (in the middle). The vertical visual field was $40^{\circ}$. The images $(30 \mathrm{~Hz}$ refresh rate) were calculated and projected at the participant's eye height. Scenes were updated interactively by a movement-tracking system that recorded the participant's motion via a cable attached to her/his waist. The visual scenes represented a one-way street $4.20 \mathrm{~m}$ wide sidewalkto-sidewalk. Traffic consisted of a motorcycle followed by two identical cars moving at a constant speed from left to right (with respect to the participant standing on the sidewalk). At the beginning of each scene, the motorcycle was $1.5 \mathrm{~s}$ away from the pedestrian and the first car was $1 \mathrm{~s}$ away from the motorcycle. The time gap between the two cars and the vehicle speed were varied.

\section{Tasks and Procedure}

Both groups participated in four sessions. First, they completed a one-hour pre-test session to assess their baseline street-crossing behavior. Approximately one week later, participants in the experimental group completed two 1.5-hour sessions of street-crossing training, and participants in the control group completed two 1.5-hour sessions of internet-use training. The training sessions were separated by approximately one week. One week after the training, participants took a one-hour post-test to assess their street-crossing behavior (immediate post-test). Six months later, their street-crossing behavior was retested (6-month post-test).

Pre and post-tests street-crossing assessments. During the pre- and post-test sessions, participants individually performed a simulated street-crossing task. On each trial, participants were asked to stand at the edge of the sidewalk, facing the experimental road. They had to look left at the visual scene, paying attention to the approaching vehicles, and decide whether or not to cross between the two cars. If they thought there was not enough time, they were to remain on the sidewalk. If they thought it was safe to cross (without running), they had to walk (at any pace) over to the sidewalk on the other side of the street. Vehicle speed (30, 40, 50, 60, and 70 $\mathrm{km} / \mathrm{hr}$ ) and time gap between the two cars ( $1 \mathrm{~s}$ to $7 \mathrm{~s}$, in $1-\mathrm{s}$ increments) were varied. The number of repetitions per time gap differed according to their probability of being considered acceptable for crossing. In fact, it has been shown that the shortest gaps are always refused and the longest gaps are always accepted (e.g., Lobjois \& Cavallo, 2007). For this reason, the 1- and 7-s time gaps were presented once, the 2- and 6-s time gaps twice, and the 3-, 4-, and 5-s time gaps, three times. This combination of 15 trials and 5 speeds resulted in a total of 75 trials. The 75 trials were randomized and divided up into 2 blocks, with a break for the participants between the blocks. The experiment started with some practical trials and lasted about 45 min.

Street-crossing training. The first training session began with a discussion about what information should be taken into account in order to cross the street safely and what safetyconscious behaviors should be adopted. The importance of speed and the impact of neglecting speed information on safety margins and accepted time gaps were explained. The participant was then asked to do three street-crossing training modules. The presentation order of the three modules was counterbalanced over the two sessions. The modules presented the same visual scenes as on the pre- and post-tests. The task was also identical. Vehicle speed (Module A: 30 vs $50 \mathrm{~km} / \mathrm{hr}$; Module B: $40 \mathrm{vs} 60 \mathrm{~km} / \mathrm{hr}$; Module C: $50 \mathrm{vs} 70 \mathrm{~km} / \mathrm{hr}$ ) and time gap (1 to $7 \mathrm{~s}$ ) were varied, making for a total of 42 randomly presented trials per module. Each module was repeated immediately, so the participant did each module twice in a row. During and after each module, 
the experimenter gave the participant two feedbacks: the safety margin, which was online computed for each trial; and the median accepted time gap, computed at the end of each module.

Internet training. Participants in the control group were given computer and internet-use training so that they could experience the same amount of social contact and have the same number of sessions as the experimental group. The control group received an introduction to the computer and instruction in how to use and access websites.

\section{Data Analysis}

Trials were scored as to whether the participant did or did not accept the available gap for crossing the street. The participant's motion (distance travelled over time) was also recorded whenever s/he decided to cross. Among the computed indicators derived from these data, the following measures are presented here:

Median accepted time gap. For each speed, the median time gap accepted by the participant was computed via a logistic regression analysis on the percentage of crossing decisions for each available time gap. This metric determined the transition point between the decision not to cross and the decision to cross (see Lobjois \& Cavallo, 2007, for more details).

Safety margin. Safety margin (SM) was the time between when the participant reached the opposite sidewalk and when the front end of the second car reached the crossing line; SM was negative if the participant was still on the road when second car passed the crossing line.

Unsafe decision. An unsafe decision was counted when the SM was less than 1.5s (c.f. Simpson, Johnston, \& Richardson, 2003). This variable was expressed as a percentage of the total number of crossings accepted by the participant.

Missed opportunity. A missed opportunity was counted when the participant refused to cross, even though based on her/his mean crossing time and initiation time, there would have been enough time to cross safely (i.e., SM would have been above $1.5 \mathrm{~s}$ ). This variable was expressed as a percentage of the total number of crossings refused by the participant.

These measures were input into ANOVAs with Group (experimental, control) as a betweenparticipant factor, and with Test Phase (pre-test, immediate post-test, and 6-month post-test) and Speed of the approaching car as within-participant factors. The significance level was set at .05. Partial $\eta^{2}$ was used as an index of the relative effect size. Significant effects were further examined using Tukey's post-hoc tests.

\section{RESULTS}

Median accepted time gap. The results showed a main effect of Group: the median time gap was larger in the experimental group (mean 3.9s; SD 0.9) than in the control group (mean 3.5s; SD $0.97), F(1,38)=5.6, p<.05, \eta^{2}=.13$. The main effect of Phase was also significant, $F(2,76)=9.7$, $\mathrm{p}<.001, \eta^{2}=.20$. The post hoc test showed that the median accepted time gap was shorter in the pre-test (mean $3.5 \mathrm{~s}$; SD 0.94) than in the immediate post-test (mean $3.82 \mathrm{~s}$; SD 0.97) and than in 
the 6-month post-test (mean $3.75 \mathrm{~s}$; SD 0.94), $\mathrm{p}<.01$ in both cases. The two post-test phases did not differ significantly ( $\mathrm{p}>$.10). Results also showed that the median accepted time gap decreased significantly as speed increased, $\mathrm{F}(4,152)=185.03, \mathrm{p}<.00001, \eta^{2}=.83$.

Safety Margins. The results indicated that SM varied between Test Phases $\mathrm{F}(2,76)=68.4$, $\mathrm{p}<.00001, \eta^{2}=.65$ : SM increased significantly between the pre-test (mean 1.53s; SD 0.69) and the immediate post-test (mean 2.12s; SD 0.81), and between the pre-test and the 6-month posttest (mean 2s; SD 0.78), $\mathrm{p}<.001$ in both cases. However, SM decreased significantly between the two post-test phases $(\mathrm{p}<.03)$. The Group $\mathrm{x}$ Phase interaction was significant $\mathrm{F}(2,76)=16.8$, $\mathrm{p}<.00001, \eta^{2}=.31$. The post-hoc test revealed that both groups exhibited a significant $\mathrm{SM}$ increase between the pre- and immediate post-tests $(\mathrm{p}<.01)$ that persisted on the 6-month posttest $(\mathrm{p}<.001)$. The participants of the experimental group had higher SM than those of the control group only in the immediate post-test $(\mathrm{p}<.01)$. Lastly, the effect of Speed was significant, $\mathrm{F}(4,152)=421.1, \mathrm{p}<.00001, \eta^{2}=.92, \mathrm{SM}$ decreasing as speed increased.

Unsafe decisions. The main effect of Phase was significant, $\mathrm{F}(2,76)=69.7, \mathrm{p}<.00001, \eta^{2}=.65$. The mean percentage of unsafe decisions was significantly higher in the pre-test (mean 50.4\%; SD 25.2) than in the immediate post-test (mean 32.1\%; SD 25.5) and than in the 6-month posttest (mean 34.5\%, SD 25.7), $\mathrm{p}<.001$ in both cases. No significant differences appeared between the two post-test phases $(\mathrm{p}>.10)$. The Group $x$ Phase interaction was significant $F(2,76)=10.9$, $\mathrm{p}<.0001, \eta^{2}=.22$, see left panel of Figure 1. The post-hoc test showed that both groups exhibited a significant decrease of unsafe decisions between the pre- and immediate post-tests $(\mathrm{p}<.001)$ that persisted on the 6 -month post-test $(\mathrm{p}<.001)$. The participants of the experimental group made fewer unsafe decisions than the participants of the control group only in the immediate post-test $(\mathrm{p}<.01)$. Lastly, the results yielded a significant main effect of Speed $\mathrm{F}(4,152)=240.1$, $\mathrm{p}<.00001, \eta^{2}=.86$, unsafe decisions increasing as speed increased.

Missed opportunities. Given the small number of missed opportunities when the car was travelling at 50,60 , or $70 \mathrm{~km} / \mathrm{hr}$, the ANOVA only pertained to speeds of 30 and $40 \mathrm{~km} / \mathrm{hr}$. The results yielded a significant main effect of Phase $F(2,76)=11.6, p<.0001, \eta^{2}=.23$. The mean percentage of missed opportunities was significantly smaller in the pre-test (mean 6.8\%; SD 9.4) than in the immediate post-test (mean 15.5\%; SD 16.2) and than in the 6-month post-test (mean $11.6 \%$; SD 14.2), $\mathrm{p}<.01$ in both cases. Missed opportunities significantly decreased between the two post-test phases $(\mathrm{p}<.05)$. The Group $\mathrm{x}$ Phase interaction was significant $\mathrm{F}(2,76)=5.8, \mathrm{p}<.01$, $\eta^{2}=.13$, see right panel of Figure 1. The post-hoc test revealed that the participants of the experimental group had higher missed opportunities than the participants of the control group only in the immediate post-test $(\mathrm{p}<.05)$. In the experimental group, we noted that missed opportunities significantly increased between the pre-test and the immediate post-test $(\mathrm{p}<.001)$, but this increase did not last until the 6-month follow-up: missed opportunities declined significantly between the immediate and 6 -month post-tests $(p<.05)$. For the control group, missed opportunities remained relatively stable over time. Lastly, the results yielded a significant main effect of Speed $F(1,38)=40.8, p<.00001, \eta^{2}=.52$, the percentage of missed opportunities decreasing as speed increased. 

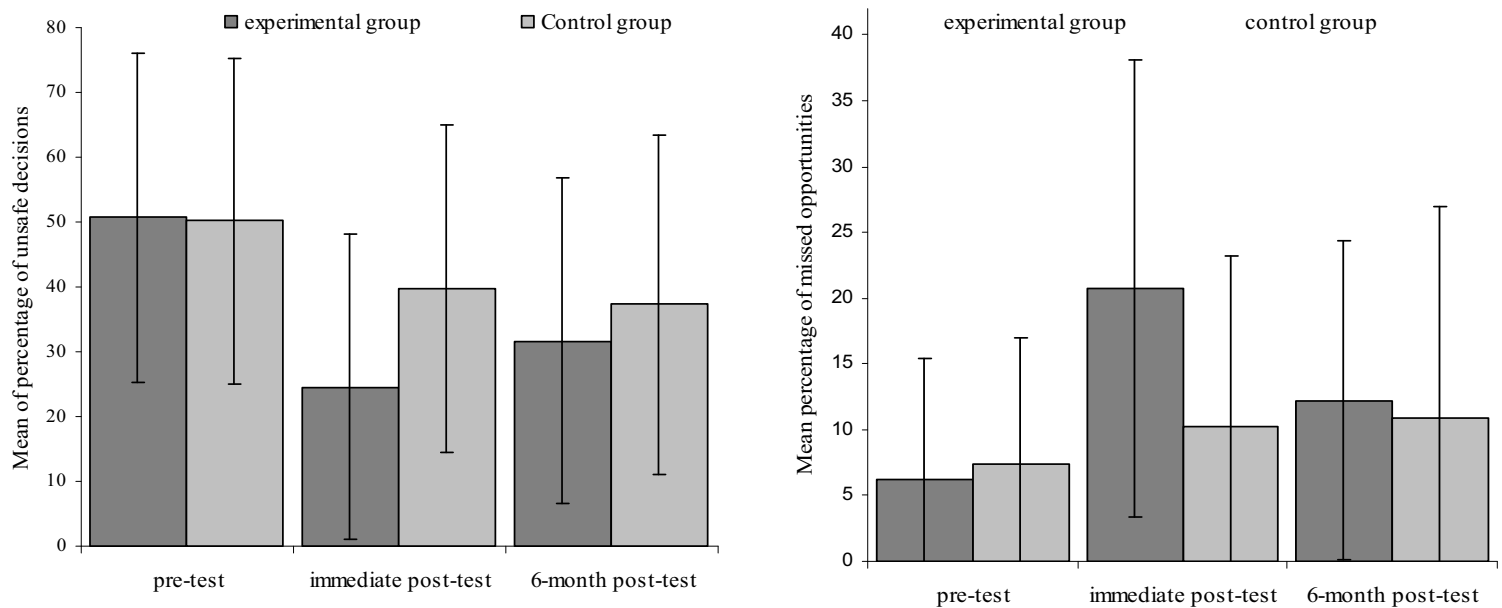

Figure 1. Mean of percentage of unsafe decisions and missed opportunities by group and testing phases. Verticals bars represent standard deviations.

\section{DISCUSSION}

This study demonstrated that a simulator-based training was able to enhance the overall safety of seniors crossing the street. Significant positive effects were found at immediate and 6-month follow-up resulting from participation in the street-crossing training program. Immediately after the training, fewer unsafe decisions were observed due to longer median accepted gaps and larger safety margins. All of the benefits were still present six months after the training. The improvements were accompanied by an increase in missed opportunities at low speeds immediately after training. However, six months later, the number of missed opportunities returned to its initial level. Notable improvements were also found in the control group.

While our results indicate a clear-cut overall improvement in street-crossing behavior, the participants still failed to make better use of the approaching vehicle's speed in their decisions and actions. The results showed a robust effect of speed on the street-crossing behaviors and decisions of the participants. Even after training, we observed that the median accepted time gap and the safety margins decreased significantly as the speed of the approaching car increased. The percentage of unsafe decisions was higher with increasing speed, in both groups. The lack of effectiveness of training in the way in which participants took into account the speed of the approaching car may reveal sensory and cognitive age-related deficits that could not be enhanced by the simulator-based training method. Elderly pedestrians seem to mainly use simplifying heuristics based on the distance of the approaching vehicle, rather than grounding their decisions on the time gap, as younger adults do (Lobjois \& Cavallo, 2007). These distance-based strategies, easier and faster to implement than strategies based on the time gap, may be the result of age-related sensory deficits, such as the decline of visual motion sensitivity (cf. e.g. Sekuler, Hutman, \& Owsley, 1980), and age-related slowing and/or weakening of cognitive processes, such as executive functions (Salthouse, Atkinson, \& Berish, 2003).

The present findings provide sufficient incentive for devoting future research to street-crossing retraining for the elderly. Further studies are required to identify the sensory, perceptual and cognitive abilities involved in street-crossing decision-making. A better understanding of these skills would be useful in designing cognitive retraining programs likely to improve the behavior of senior pedestrians, as in Roenker et al.'s (2003) program for elderly drivers. 


\section{REFERENCES}

Cavallo, V., Lobjois, R., \& Vienne, F. (2006). The interest of an interactive road crossing simulation for the study of adaptive road crossing behaviour. Proceedings of the First Driving simulation conference Asia-Pacific, Tsukuba, Japan.

Espié, S. (1999). Vehicle-driven simulator versus traffic-driven simulator: The INRETS approach. Proceeding of the First Driving Simulation Conference Europe. Paris, 367-376.

Kua, A., Korner-Bitensky, N., Desrosiers, J., Man-Son-Hing, M., \& Marshall, S. (2007). Older driver retraining: a systematic review of effectiveness. Journal of Safety Research, 38, 81-90.

Lobjois R., \& Cavallo, V. (2007). Age-related differences in street-crossing decisions: The effects of vehicule speed and time constraints on gap selection in an estimation task, Accident Analysis and Prevention, 39, 934-943.

National Highway Traffic Safety Administration (NHTSA), 2001. Traffic Safety Facts 2000: Pedestrians. Reports No. DOT-HS-809311. US Department of Transport, Washington, DC.

McCoy, P.T., Tarawneh, M.S., Bishu, R.R., Ashman, R.D., \& Foster, B.G. (1993). Evaluation of countermeasures for improving driving performance of older drivers. Transportation Research Record, 1405, 72-80.

Observatoire National Interministériel de Sécurité Routière (ONISR) (2006). Grand thèmes de la sécurité routière en France : Piétons. ONISR.

Ostrow, A.C., Shaffron, P. \& McPherson, K. (1992). The effects of a joint range-of-motion physical fitness training program on the automobile driving skills of older adults. Journal of Safety Research, 23, 207-219.

Owsley, C., Stalvey, B.T., \& Phillips, J.M. (2003). The efficacy of an educational intervention in promoting self-regulation among high-risk older drivers. Analysis and Prevention, 35, 393400 .

Oxley, J., Fildes, B., Ihsen, E., Charlton, J. \& Day, R. (2005). Crossing roads safely: an experimental study of age differences in gap selection by pedestrians. Accident Analysis and Prevention, 37, 962-971.

Roenker, D.L., Cissel G.M., Ball, K.K., Wadley, V.G., \& Edwards J.D. (2003). Speed-ofprocessing and driving simulator training result in improved driving performance, Human Factors, 45, 218-233.

Salthouse, T.A., Atkinson, T.M. \& Berish, D.E. (2003). Executive functioning as a potential mediator of age-related cognitive decline in normal adults. Journal of Experimental Psychology, 4, 566-594.

Sekuler, R., Hutman, L.P., \& Owsley, C.J. (1980). Human aging and spatial vision. Science, 209, 1255-1256.

Simpson, G., Johnston, L., \& Richardson, M. (2003). An investigation of road crossing in a virtual environment. Accident Analysis and Prevention, 35, 787-796. 